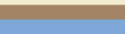

Noberef tuluer Journal of Nonlinear Mathematical Physics

\title{
On the Discretization of the Coupled Integrable Dispersionless Equations
}

Luc Vinet, Guo-Fu Yu

To cite this article: Luc Vinet, Guo-Fu Yu (2013) On the Discretization of the Coupled Integrable Dispersionless Equations, Journal of Nonlinear Mathematical Physics 20:1, 106-125, DOI: https://doi.org/10.1080/14029251.2013.792476

To link to this article: https://doi.org/10.1080/14029251.2013.792476

Published online: 04 January 2021 


\title{
On the Discretization of the Coupled Integrable Dispersionless Equations
}

\author{
Luc Vinet \\ Centre de Recherches Mathématiques, Université de Montréal, \\ C.P.6128, Centre-ville Station, Montréal, Québec, H3C 3J7, Canada \\ luc.vinet@umontreal.ca \\ Guo-Fu Yu \\ Department of Mathematics, Shanghai Jiao Tong University, \\ Shanghai 200240, P.R. China \\ Centre de Recherches Mathématiques, Université de Montréal, \\ C.P.6128, Centre-ville Station, Montréal, Québec, H3C 3J7, Canada \\ gfyu@sjtu.edu.cn \\ Received 4 August 2012 \\ Accepted 18 January 2013
}

\begin{abstract}
We study the integrable discretization of the coupled integrable dispersionless equations. Two semi-discrete version and one full-discrete version of the system are given via Hirota's bilinear method. Soliton solutions for the derived discrete systems are also presented.
\end{abstract}

Keywords: Soliton solution; Integrable discretization; Hirota bilinear method.

2000 Mathematics Subject Classification: 37K10, 35C05, 37K40

\section{Introduction}

The activity in the field of partial difference integrable equations based on bilinear form was initiated by Hirota [6-9] who proposed discrete analogues of the KdV, the Toda chain, the sine-Gordon (SG) equations, etc. (actually of almost all interesting soliton equations). Another powerful recipe in this aspect is the method based on Lax pair $[1,28]$. The bilinear integrable discretization method is based on the bilinear formalism and follows three steps. First a given differential equation is transformed into a bilinear equation by a dependent variable transformation. Second the bilinear equation is discretized using the gauge-invariance of the bilinear equation and the integrability of the discretized bilinear equation is determined through soliton solutions. Third the discrete bilinear equation is transformed into a discrete nonlinear equation in the ordinary form by an associated dependent variable transformation. Based on bilinear forms and determinant structure of solutions, Hirota's discretization method has been developed to construct discrete versions of $(2+1)$-dimensional sinhGordon equaiton [17], two dimensional Leznov lattice equaiton [18], Camassa-Holm equation [26], short pulse equation [5] and so on.

The already known fully discrete integrable systems are rare, only fully discrete KP (or HirotaMiwa) equation and fully discrete BKP equation and so on. So it would be meaningful to use 
Hirota's discretization method to search for more integrable fully discrete systems and research their integrable properties. These discrete versions may have substantial applications or their solutions have beautiful mathematical structures.

In this paper we consider the coupled integrable dispersionless (CID) equations [21]

$$
\begin{aligned}
& q_{t}+2 r r_{x}=0, \\
& r_{x t}-2 q r=0,
\end{aligned}
$$

where $q$ and $r$ are both functions of $x$ and $t$, the subscripts denote derivatives. The inverse scattering scheme of eqs. (1.1) and its compatibility condition are given as

$$
V_{x}=U V, \quad V_{t}=W V,
$$

and

$$
U_{t}-W_{x}+[U, W]=0
$$

where

$$
\begin{aligned}
& U=-\mathrm{i} \lambda\left(\begin{array}{ll}
q_{x} & r_{x} \\
r_{x} & -q_{x}
\end{array}\right), \\
& W=\left(\begin{array}{cc}
0 & -r \\
r & 0
\end{array}\right)+\frac{\mathrm{i}}{2 \lambda}\left(\begin{array}{cc}
1 & 0 \\
0 & -1
\end{array}\right) .
\end{aligned}
$$

A generalized inverse scheme of the CID system was proposed from the group theoretical point of view in [19]. Eqs. (1.1) describe the current-fed string within an external magnetic field. The detailed physical application of the system (1.1) was stated in [20].

It should also be noted that the CID equations are related to the sine-Gordon equation

$$
\phi_{x t}=2 q_{0} \sin \phi
$$

through the variable transformation [15]

$$
q=q_{0} \cos \phi, \quad r=\frac{1}{2} \phi_{t} .
$$

In [22], a slightly general result between CID equations and the sine-Gordon equation has been presented. The integrability and hidden symmetry of the CID equations are analyzed in [33]. The $N$ soliton solutions and the infinitely many conservation laws are studied in [29,31]. Painlevé property and Bäcklund transformation are shown in $[3,4]$. In the following we present two remarks on the CID system (1.1).

Remark 1.1. Although several papers called the system the coupled integrable dispersionless equations, this system is not a dispersionless system.

Remark 1.2. This system was already recognized by many people before Konno and Oono. As Hirota and Tsujimoto claimed, this system is related to the sine-Gordon equation. This was already known since sine-Gordon equation was derived from this system in the AKNS formulation, see page 13, [2].

Since the physical application of the CID equations, it would be meaningful to consider its integrable discrete versions. The purpose of this paper is to construct discrete analogues of the 
CID equations by Hirota's discretization approach. We present two gauge invariant semi-discrete CID equations and one fully discrete CID equations. The soliton solutions are deduced using the perturbation method.

The paper is organized as follows: In section 2, we give a $x$-discrete version of the CID equations and present the soliton solutions. In section 3 , we derive a $t$-discrete version of CID equations. In section 4, a fully discrete CID version is worked out. In section 5, discrete analogues and Casorati determinant solutions are given by 2-reduction technique. The section 6 is devoted to conclusion and discussions.

\section{Integrable semi-discrete version in $\mathrm{x}$ direction}

Through the dependent variable transformation

$$
q=1-(\ln F)_{x t}, \quad r=G / F,
$$

the CID equations (1.1) can be transformed into the bilinear form

$$
\begin{aligned}
& D_{t}^{2} F \cdot F-2 G^{2}=0, \\
& \left(D_{x} D_{t}-2\right) G \cdot F=0 .
\end{aligned}
$$

It is easy to see that this bilinear form is just the 1-component form of the nonlinear coupled KleinGordon (sine-Gordon) equation [13]. Bilinear eqs. (2.2) and (2.3) are transformed into

$$
\begin{aligned}
& D_{t}^{2} F \cdot F=2 G^{2}, \\
& \left(D_{x} D_{t}-1\right) G \cdot F=0,
\end{aligned}
$$

through scaling transformation $x \rightarrow \frac{1}{2} x$. Eqs. (2.4)-(2.5) are bilinear forms of the coupled nonlinear Klein-Gordon equation [16]. Let

$$
\begin{aligned}
& F=f^{*} f, \\
& G=i D_{t} f^{*} \cdot f .
\end{aligned}
$$

Then $F$ and $G$ satisfy the bilinear eqs. (2.4)-(2.5) provided that $f$ and $f^{*}$ satisfy

$$
\begin{aligned}
& \left(D_{x} D_{t}^{2}-D_{t}\right) f^{*} \cdot f=0, \\
& D_{t}^{2} f^{*} \cdot f=0,
\end{aligned}
$$

which is a bilinear form of the sine-Gordon equation [16].

The coupled differential-difference CID equations are obtained by discretizing the spacial part of the bilinear eqs. (2.2)-(2.3),

$$
D_{x} G \cdot F \rightarrow \frac{1}{\varepsilon}\left(G_{n+1} F_{n}-G_{n} F_{n+1}\right),
$$

where $x=n \varepsilon, n$ being integers and $\varepsilon$ a spatial-interval. We obtain

$$
\begin{aligned}
& D_{t} G_{n+1} \cdot F_{n}-D_{t} G_{n} \cdot F_{n+1}-2 \varepsilon G_{n} F_{n}=0, \\
& D_{t}^{2} F_{n} \cdot F_{n}-2 G_{n}^{2}=0 .
\end{aligned}
$$


We postulate that the discretized bilinear forms are invariant under the gauge transformation:

$$
\begin{aligned}
& F_{n} \rightarrow F_{n} \exp \left(q_{0} n\right), \\
& G_{n} \rightarrow G_{n} \exp \left(q_{0} n\right) .
\end{aligned}
$$

We find a gauge invariant semi-discrete bilinear CID equations

$$
\begin{aligned}
& D_{t} G_{n+1} \cdot F_{n}-D_{t} G_{n} \cdot F_{n+1}-\varepsilon\left(G_{n+1} F_{n}+G_{n} F_{n+1}\right)=0, \\
& D_{t}^{2} F_{n} \cdot F_{n}-2 G_{n}^{2}=0 .
\end{aligned}
$$

Let $G_{n}=r_{n} F_{n}, q_{n}=\left(\ln F_{n}\right)_{t}$. Then eqs. (2.15)-(2.16) are transformed into

$$
\begin{aligned}
& r_{n+1, t}-r_{n, t}+\left(q_{n+1}-q_{n}-\varepsilon\right)\left(r_{n+1}+r_{n}\right)=0, \\
& q_{n, t}=r_{n}^{2} .
\end{aligned}
$$

When we take the continuum limit $\varepsilon \rightarrow 0$, (2.17)-(2.18) reduce to

$$
\begin{aligned}
& r_{x t}-2\left(1-q_{x}\right) r=0, \\
& q_{t}=r^{2} .
\end{aligned}
$$

Through transformation $1-q_{x} \rightarrow q$, the above nonlinear system can be transformed into the CID eqs. (1.1). So we can regard (2.17)-(2.18) as a semi-discrete version of the CID equation. In the following discussion we take $\varepsilon=1$ in eq. (2.15) for simplicity. According to the Hirota method, in order to construct soliton solutions, we expand $G$ and $F$ in series with a small parameter $\varepsilon$ as

$$
\begin{aligned}
& F_{n}=1+\varepsilon^{2} F_{n}^{(2)}+\varepsilon^{4} F_{n}^{(4)}+\cdots+\varepsilon^{2 k} F_{n}^{(2 k)}+\cdots \\
& G_{n}=\varepsilon G_{n}^{(1)}+\varepsilon^{3} G_{n}^{(3)}+\cdots+\varepsilon^{(2 k+1)} G_{n}^{(2 k+1)}+\cdots
\end{aligned}
$$

Substituting the expansion into the above bilinear eqs. (2.15)-(2.16) we find that there are only odd order terms of $\varepsilon$ in the first equation while only even order terms appear in the second one. Comparing the coefficients at each order of $\varepsilon$, we have

$$
\begin{aligned}
\varepsilon: & G_{n+1, t}^{(1)}-G_{n, t}^{(1)}-G_{n+1}^{(1)}-G_{n}^{(1)}=0, \\
\varepsilon^{2}: & D_{t}^{2}\left(F_{n}^{(2)} \cdot 1+1 \cdot F_{n}^{(2)}\right)=2\left(G_{n}^{(1)}\right)^{2}, \\
\varepsilon^{3}: & D_{t}\left[G_{n+1}^{(1)} \cdot F_{n}^{(2)}+G_{n+1}^{(3)} \cdot 1-G_{n}^{(1)} \cdot F_{n+1}^{(2)}-G_{n}^{(3)} \cdot 1\right] \\
& =G_{n+1}^{(3)}+G_{n}^{(3)}+G_{n+1}^{(1)} F_{n}^{(2)}+G_{n}^{(1)} F_{n+1}^{(2)}, \\
\varepsilon^{4}: & 2 D_{t}^{2} F_{n}^{(4)} \cdot 1+D_{t}^{2} F_{n}^{(2)} \cdot F_{n}^{(2)}=4 G_{n}^{(1)} G_{n}^{(3)},
\end{aligned}
$$

By solving the above linear system, we obtain the one-soliton solution

$$
G_{n}=\varepsilon \exp \left(\eta_{1}\right), \quad F_{n}=1+\varepsilon^{2} \frac{1}{4 \alpha_{1}^{2}} \exp \left(2 \eta_{1}\right)
$$


where $\eta_{1}=\alpha_{1} t+\beta_{1} n+\gamma_{1}$ and $\alpha_{1}=\operatorname{coth}\left(\beta_{1} / 2\right) . \alpha_{1}, \gamma_{1}$ are arbitrary constants, and the two-soliton solution:

$$
\begin{aligned}
G_{n}=\varepsilon & {\left[\exp \left(\eta_{1}\right)+\exp \left(\eta_{2}\right)\right]+\varepsilon^{3}\left[A \exp \left(2 \eta_{1}+\eta_{2}\right)+B \exp \left(\eta_{1}+2 \eta_{2}\right)\right], } \\
F_{n}=1+ & \varepsilon^{2}\left[\frac{1}{4 \alpha_{1}^{2}} \exp \left(2 \eta_{1}\right)+\frac{1}{4 \alpha_{2}^{2}} \exp \left(2 \eta_{2}\right)+\frac{2}{\left(\alpha_{1}+\alpha_{2}\right)^{2}} \exp \left(\eta_{1}+\eta_{2}\right)\right] \\
& +\varepsilon^{4} C \exp \left(2 \eta_{1}+2 \eta_{2}\right),
\end{aligned}
$$

where

$$
A=\frac{\left(\alpha_{1}-\alpha_{2}\right)^{2}}{4 \alpha_{1}^{2}\left(\alpha_{1}+\alpha_{2}\right)^{2}}, \quad B=\frac{\left(\alpha_{1}-\alpha_{2}\right)^{2}}{4 \alpha_{2}^{2}\left(\alpha_{1}+\alpha_{2}\right)^{2}}, \quad C=\frac{\left(\alpha_{1}-\alpha_{2}\right)^{4}}{16 \alpha_{1}^{2} \alpha_{2}^{2}\left(\alpha_{1}+\alpha_{2}\right)^{4}}
$$

The corresponding plots are shown in Fig. 1. We can use the following compact expression for the above two-soliton solution,

$$
\begin{aligned}
F_{n}= & 1+a\left(1,1^{*}\right) \exp \left(\eta_{1}+\eta_{1}^{*}\right)+a\left(1,2^{*}\right) \exp \left(\eta_{1}+\eta_{2}^{*}\right) \\
& +a\left(2,1^{*}\right) \exp \left(\eta_{2}+\eta_{1}^{*}\right)+a\left(2,2^{*}\right) \exp \left(\eta_{2}+\eta_{2}^{*}\right) \\
& +a\left(1,2,1^{*}, 2^{*}\right) \exp \left(\eta_{1}+\eta_{2}+\eta_{1}^{*}+\eta_{2}^{*}\right), \\
G_{n}= & \exp \left(\eta_{1}\right)+\exp \left(\eta_{2}\right)+a\left(1,2,1^{*}\right) \exp \left(\eta_{1}+\eta_{2}+\eta_{1}^{*}\right) \\
& +a\left(1,2,2^{*}\right) \exp \left(\eta_{1}+\eta_{2}+\eta_{2}^{*}\right),
\end{aligned}
$$

where $\eta_{i}^{*}=\eta_{i},(i=1,2)$ and

$$
\begin{aligned}
& a(i, j)=\left(\alpha_{i}-\alpha_{j}\right)^{2}, \\
& a\left(i, j^{*}\right)=\frac{1}{\left(\alpha_{i}+\alpha_{j}\right)^{2}}, \\
& a\left(i^{*}, j^{*}\right)=\left(\alpha_{i}-\alpha_{j}\right)^{2}, \\
& a\left(i, j, k^{*}\right)=a(i, j) a\left(i, k^{*}\right) a\left(j, k^{*}\right), \\
& a\left(i, j, k^{*}, l^{*}\right)=a(i, j) a\left(i, k^{*}\right) a\left(i, l^{*}\right) a\left(j, k^{*}\right) a\left(j, l^{*}\right) a\left(k^{*}, l^{*}\right) .
\end{aligned}
$$


In the same way, we can construct the three-soliton solution,

$$
\begin{aligned}
G_{n}= & \exp \left(\eta_{1}\right)+\exp \left(\eta_{2}\right)+\exp \left(\eta_{3}\right) \\
& +a\left(1,2,1^{*}\right) \exp \left(2 \eta_{1}+\eta_{2}\right)+a\left(1,3,1^{*}\right) \exp \left(2 \eta_{1}+\eta_{3}\right)+a\left(2,3,2^{*}\right) \exp \left(2 \eta_{2}+\eta_{3}\right) \\
& +a\left(1,2,2^{*}\right) \exp \left(\eta_{1}+2 \eta_{2}\right)+a\left(1,3,3^{*}\right) \exp \left(\eta_{1}+2 \eta_{3}\right)+a\left(2,3,3^{*}\right) \exp \left(\eta_{2}+2 \eta_{3}\right) \\
& +\left[a\left(1,2,3^{*}\right)+a\left(1,3,2^{*}\right)+a\left(2,3,1^{*}\right)\right] \exp \left(\eta_{1}+\eta_{2}+\eta_{3}\right) \\
& +a\left(1,2,3,1^{*} 2^{*}\right) \exp \left(2 \eta_{1}+2 \eta_{2}+\eta_{3}\right)+a\left(1,2,3,1^{*} 3^{*}\right) \exp \left(2 \eta_{1}+\eta_{2}+2 \eta_{3}\right) \\
& +a\left(1,2,3,2^{*}, 3^{*}\right) \exp \left(\eta_{1}+2 \eta_{2}+2 \eta_{3}\right) \\
F_{n}= & +a\left(1,1^{*}\right) \exp \left(2 \eta_{1}\right)+a\left(2,2^{*}\right) \exp \left(2 \eta_{2}\right)+a\left(3,3^{*}\right) \exp \left(2 \eta_{3}\right) \\
& +\left[a\left(1,2^{*}\right)+a\left(2,1^{*}\right)\right] \exp \left(\eta_{1}+\eta_{2}\right)+\left[a\left(2,3^{*}\right)+a\left(3,2^{*}\right)\right] \exp \left(\eta_{2}+\eta_{3}\right) \\
& +\left[a\left(1,3^{*}\right)+a\left(3,1^{*}\right)\right] \exp \left(\eta_{1}+\eta_{3}\right) \\
& +a\left(1,2,1^{*}, 2^{*}\right) \exp \left(2 \eta_{1}+2 \eta_{2}\right)+a\left(1,3,1^{*}, 3^{*}\right) \exp \left(2 \eta_{1}+2 \eta_{3}\right)+a\left(2,3,2^{*}, 3^{*}\right) \exp \left(2 \eta_{2}+2 \eta_{3}\right) \\
& +\left[a\left(1,2,1^{*}, 3^{*}\right)+a\left(1,3,1^{*}, 2^{*}\right)\right] \exp \left(2 \eta_{1}+\eta_{2}+\eta_{3}\right) \\
& +\left[a\left(1,2,2^{*}, 3^{*}\right)+a\left(2,3,1^{*}, 2^{*}\right)\right] \exp \left(\eta_{1}+2 \eta_{2}+\eta_{3}\right) \\
& +\left[a\left(1,3,2^{*}, 3^{*}\right)+a\left(2,3,1^{*}, 3^{*}\right)\right] \exp \left(\eta_{1}+\eta_{2}+2 \eta_{3}\right) \\
& +a\left(1,2,3,1^{*}, 2^{*}, 3^{*}\right) \exp \left(2 \eta_{1}+2 \eta_{2}+2 \eta_{3}\right)
\end{aligned}
$$

where the coefficients are defined as (2.33)-(2.37) and

$$
\begin{aligned}
a\left(i, j, k, l^{*}, m^{*}, n^{*}\right)= & a(i, j) a(i, k) a\left(i, l^{*}\right) a\left(i, m^{*}\right) a\left(i, n^{*}\right) \\
& \times a(j, k) a\left(j, l^{*}\right) a\left(j, m^{*}\right) a\left(j, n^{*}\right) \\
& \times a\left(k, l^{*}\right) a\left(k, m^{*}\right) a\left(k, n^{*}\right) \\
& \times a\left(l^{*}, m^{*}\right) a\left(l^{*}, n^{*}\right) \\
& \times a\left(m^{*}, n^{*}\right) .
\end{aligned}
$$

The above expression of multi-soliton solutions suggest the exact $N$-soliton solutions of eqs. (2.15)(2.16) in the following form

$$
\begin{aligned}
& F_{n}=\sum_{\mu=0,1}^{(e)} \exp \left[\sum_{j=1}^{2 N} \mu_{j} \eta_{j}+\sum_{1 \leq k<l}^{2 N} \mu_{k} \mu_{l} A_{k l}\right] \\
& G_{n}=\sum_{v=0,1}^{(o)} \exp \left[\sum_{j=1}^{2 N} v_{j} \eta_{j}+\sum_{1 \leq k<l}^{2 N} v_{k} v_{l} A_{k l}\right]
\end{aligned}
$$

where

$$
\begin{aligned}
& \eta_{j}=\eta_{N+j}=\alpha_{j} t+\beta_{j} n+\gamma_{j}, \quad \alpha_{j}=\operatorname{coth}\left(\beta_{j} / 2\right), \quad j=1,2, \cdots, N \\
& \exp \left(A_{k l}\right)=\left(\alpha_{k}-\alpha_{l}\right)^{2}, \quad k<l=2,3, \cdots, N \\
& \exp \left(A_{k, N+l}\right)=\frac{1}{\left(\alpha_{k}+\alpha_{l}\right)^{2}}, \quad k, l=1,2, \cdots, N \\
& \exp \left(A_{N+k, N+l}\right)=\left(\alpha_{k}-\alpha_{l}\right)^{2}, \quad k<l=2,3, \cdots, N
\end{aligned}
$$

Here $\alpha_{j}, \gamma_{j}$ are both real parameters relating respectively to the amplitude and phase of the $i$ th soliton. The sum $\sum_{\mu=0,1}^{(e)}$ indicates the summation over all possible combinations of $\mu_{1}=0,1, \mu_{2}=$ 
$0,1, \cdots, \mu_{2 N}=0,1$, under the condition

$$
\sum_{j=1}^{N} \mu_{j}=\sum_{j=1}^{N} \mu_{N+j}
$$

and $\sum_{v=0,1}^{(o)}$ indicates the summation over all possible combinations of $v_{1}=0,1, v_{2}=0,1, \cdots, v_{2 N}=$ 0,1 , under the condition

$$
\sum_{j=1}^{N} v_{j}=1+\sum_{j=1}^{N} v_{N+j}
$$

The form of the $N$-soliton solution (2.41)-(2.42) is the same as that of the combined SchrödingermKdV equation in the Ref. [10]. The proof of the $N$-soliton solution here can be completed by induction and it is similar as that in [10]. One can check the details therein.

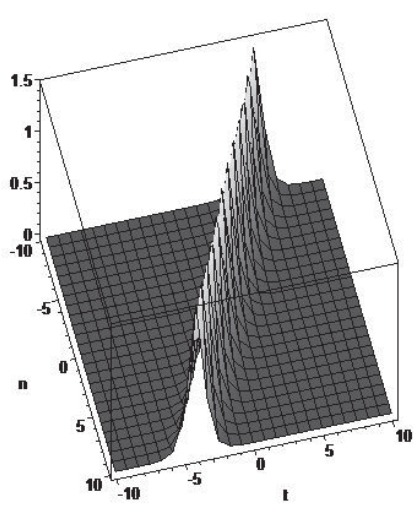

(a)

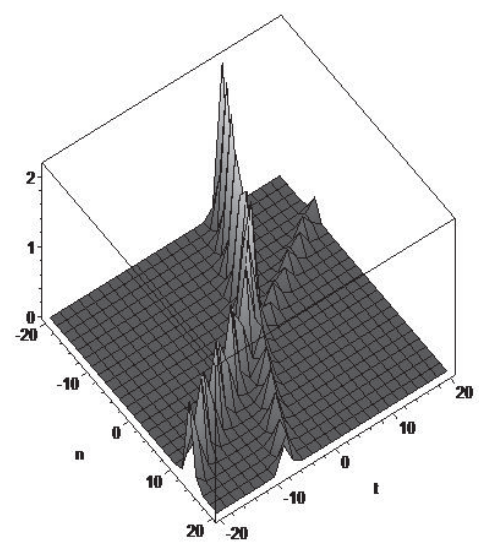

(b)

Fig. 1. soliton solution of $r\left(\beta_{1}=1, \beta_{2}=2, \gamma_{1}=\gamma_{2}=0\right)$ : $\quad(a)$ one-soliton, (b) two-soliton

\section{Integrable semi-discrete version in t direction}

A coupled differential-difference CID equations are obtained by discretizing the time part of the bilinear eqs. (2.2)-(2.3),

$$
\begin{aligned}
& D_{t} G \cdot F \rightarrow \frac{1}{\delta}\left(G_{m+1} F_{m}-G_{m} F_{m+1}\right), \\
& D_{t}^{2} F \cdot F \rightarrow \frac{2}{\delta^{2}}\left(F_{m+1} F_{m-1}-\left(F_{m}\right)^{2}\right),
\end{aligned}
$$

where $t=m \delta, m$ is an integer and $\delta$ a time-interval. We find a gauge invariant set of semi-discrete bilinear CID equations

$$
\begin{aligned}
& D_{x} G_{m+1} \cdot F_{m}-D_{x} G_{m} \cdot F_{m+1}=\delta\left(F_{m} G_{m+1}+F_{m+1} G_{m}\right), \\
& F_{m+1} F_{m-1}-F_{m}^{2}=\delta^{2} G_{m}^{2} .
\end{aligned}
$$


Let $G_{m}=r_{m} F_{m}, u_{m}=\ln F_{m}$. Eqs. (3.3)-(3.4) are then transformed into the ordinary nonlinear form

$$
\begin{aligned}
& r_{m+1, x}-r_{m, x}+\left(r_{m+1}+r_{m}\right)\left(u_{m+1, x}-u_{m, x}\right)=\delta\left(r_{m}+r_{m+1}\right), \\
& e^{u_{m+1}+u_{m-1}-2 u_{m}}-1=\delta^{2} r_{m}^{2} .
\end{aligned}
$$

When we take the continuum limit $\delta \rightarrow 0$, eqs. (3.5)-(3.6) reduce to

$$
\begin{aligned}
& r_{x t}+2 r u_{x t}=2 r, \\
& u_{t t}=r^{2}
\end{aligned}
$$

Performing the variable transformation $q=1-u_{x t}$ and differentiating eq. (3.8) with $x$, we obtain the original nonlinear eqs. (1.1). As before we take $\delta=1$ for simplicity in the following. Using the perturbation method, we can construct soliton solutions of the semi-discrete CID eqs. (3.3)-(3.4). For the one-soliton solution we have

$$
G_{m}=\exp \left(\xi_{1}\right), \quad F_{m}=1+\frac{\left(\alpha_{1}^{2}-1\right)^{2}}{16 \alpha_{1}^{2}} \exp \left(2 \xi_{1}\right)
$$

where $\xi_{i}=\alpha_{i} x+\beta_{i} m+\gamma_{i}$ and $\alpha_{i}=\operatorname{coth}\left(\beta_{i} / 2\right) . \alpha_{i}, \gamma_{i}$ are arbitrary constants. The two-soliton solution reads

$$
\begin{aligned}
G_{m}= & \exp \left(\xi_{1}\right)+\exp \left(\xi_{2}\right)+A \exp \left(2 \xi_{1}+\xi_{2}\right)+B \exp \left(\xi_{1}+2 \xi_{2}\right), \\
F_{m}= & 1+\frac{\left(\alpha_{1}^{2}-1\right)^{2}}{16 \alpha_{1}^{2}} \exp \left(2 \xi_{1}\right)+\frac{\left(\alpha_{2}^{2}-1\right)^{2}}{16 \alpha_{2}^{2}} \exp \left(2 \xi_{2}\right) \\
& +\frac{\alpha_{1}^{2} \alpha_{2}^{2}-\alpha_{1}^{2}-\alpha_{2}^{2}+1}{2\left(\alpha_{1}+\alpha_{2}\right)^{2}} \exp \left(\xi_{1}+\xi_{2}\right)+C \exp \left(2 \xi_{1}+2 \xi_{2}\right),
\end{aligned}
$$

where

$$
\begin{aligned}
& A=\frac{\left(\alpha_{1}^{2}-1\right)^{2}\left(\alpha_{1}-\alpha_{2}\right)^{2}}{16 \alpha_{1}^{2}\left(\alpha_{1}+\alpha_{2}\right)^{2}}, \quad B=\frac{\left(\alpha_{2}^{2}-1\right)^{2}\left(\alpha_{1}-\alpha_{2}\right)^{2}}{16 \alpha_{2}^{2}\left(\alpha_{1}+\alpha_{2}\right)^{2}}, \\
& C=\frac{\left(\alpha_{1}^{2}-1\right)^{2}\left(\alpha_{2}^{2}-1\right)^{2}\left(\alpha_{1}-\alpha_{2}\right)^{4}}{256 \alpha_{1}^{2} \alpha_{2}^{2}\left(\alpha_{1}+\alpha_{2}\right)^{4}} .
\end{aligned}
$$

Similar as the previous section, we can rewrite the two-soliton solution as the following compact form

$$
\begin{aligned}
G_{m}= & \exp \left(\xi_{1}\right)+\exp \left(\xi_{2}\right)+b\left(1,2,1^{*}\right) \exp \left(2 \xi_{1}+\xi_{2}\right)+b\left(1,2,2^{*}\right) \exp \left(\xi_{1}+2 \xi_{2}\right), \\
F_{m}= & 1+b\left(1,1^{*}\right) \exp \left(2 \xi_{1}\right)+b\left(2,2^{*}\right) \exp \left(2 \xi_{2}\right) \\
& +\left[b\left(1,2^{*}\right)+b\left(2,1^{*}\right)\right] \exp \left(\xi_{1}+\xi_{2}\right)+b\left(1,2,1^{*}, 2^{*}\right) \exp \left(2 \xi_{1}+2 \xi_{2}\right),
\end{aligned}
$$


where

$$
\begin{aligned}
& b(k, l)=\frac{4\left(\alpha_{k}-\alpha_{l}\right)^{2}}{\alpha_{k}^{2} \alpha_{l}^{2}-\alpha_{k}^{2}-\alpha_{l}^{2}+1}, \\
& b\left(k, l^{*}\right)=\frac{\alpha_{k}^{2} \alpha_{l}^{2}-\alpha_{k}^{2}-\alpha_{l}^{2}+1}{4\left(\alpha_{k}+\alpha_{l}\right)^{2}}, \\
& b\left(k^{*}, l^{*}\right)=\frac{4\left(\alpha_{k}-\alpha_{l}\right)^{2}}{\alpha_{k}^{2} \alpha_{l}^{2}-\alpha_{k}^{2}-\alpha_{l}^{2}+1}, \\
& b\left(i, j, k^{*}\right)=b(i, j) b\left(i, k^{*}\right) b\left(j, k^{*}\right), \\
& b\left(i, j, k^{*}, l^{*}\right)=b(i, j) b\left(i, k^{*}\right) b\left(i, l^{*}\right) b\left(j, k^{*}\right) b\left(j, l^{*}\right) b\left(k^{*}, l^{*}\right) .
\end{aligned}
$$

Three soliton solution is presented by the expression (2.38)-(2.39) with the coefficients $a(\cdots)$ substituted by $b(\cdots)$ which obey (3.15)-(3.19) and operation rule (2.37). Generally, the $N$-soliton solution is given by

$$
\begin{aligned}
& F_{m}=\sum_{\mu=0,1}^{(e)} \exp \left[\sum_{j=1}^{2 N} \mu_{j} \xi_{j}+\sum_{1 \leq k<l}^{2 N} \mu_{k} \mu_{l} A_{k l}\right], \\
& G_{m}=\sum_{v=0,1}^{(o)} \exp \left[\sum_{j=1}^{2 N} v_{j} \xi_{j}+\sum_{1 \leq k<l}^{2 N} v_{k} v_{l} A_{k l}\right],
\end{aligned}
$$

where

$$
\begin{aligned}
& \eta_{j}=\xi_{N+j}=\alpha_{j} x+\beta_{j} m+\gamma_{j}, \quad \exp \left(\beta_{j}\right)=\frac{\alpha_{j}+1}{\alpha_{j}-1}, \quad j=1,2, \cdots, N, \\
& \exp \left(A_{k l}\right)=\frac{4\left(\alpha_{k}-\alpha_{l}\right)^{2}}{\alpha_{k}^{2} \alpha_{l}^{2}-\alpha_{k}^{2}-\alpha_{l}^{2}+1}, k<l=2,3, \cdots, N \\
& \exp \left(A_{k, N+l}\right)=\frac{\alpha_{k}^{2} \alpha_{l}^{2}-\alpha_{k}^{2}-\alpha_{l}^{2}+1}{4\left(\alpha_{k}+\alpha_{l}\right)^{2}}, k, l=1,2, \cdots, N, \\
& \exp \left(A_{N+k, N+l}\right)=\frac{4\left(\alpha_{k}-\alpha_{l}\right)^{2}}{\alpha_{k}^{2} \alpha_{l}^{2}-\alpha_{k}^{2}-\alpha_{l}^{2}+1}, k<l=2,3, \cdots, N .
\end{aligned}
$$

Here $\alpha_{j}, \beta_{j}, \gamma_{j}$ are all real parameters. The summations $\sum_{\mu=0,1}^{(e)}$ and $\sum_{v=0,1}^{(o)}$ satisfy the condition (2.47) and (2.48) respectively. The proof of $N$-soliton solution is similar as that in [10].

\section{Integrable fully discrete version of the CID equations}

The fully discrete CID equations are obtained by discretizing the bilinear eqs. (2.2)-(2.3)

$$
\begin{aligned}
& D_{t}^{2} F \cdot F \rightarrow \frac{2}{\varepsilon^{2}}\left(F_{n}^{m+1} F_{n}^{m-1}-\left(F_{n}^{m}\right)^{2}\right), \\
& D_{x} D_{t} G \cdot F \rightarrow \frac{1}{\delta \varepsilon}\left(G_{n+1}^{m+1} F_{n}^{m}-G_{n}^{m+1} F_{n+1}^{m}-G_{n+1}^{m} F_{n}^{m+1}+G_{n}^{m} F_{n+1}^{m+1}\right) .
\end{aligned}
$$

In the following, we also postulate that the discretized bilinear forms are invariant under the gauge transformation:

$$
\begin{aligned}
& F_{n}^{m} \rightarrow F_{n}^{m} \exp \left(q_{0} n+p_{0} m\right), \\
& G_{n}^{m} \rightarrow G_{n}^{m} \exp \left(q_{0} n+p_{0} m\right) .
\end{aligned}
$$


We rewrite $G F$ as

$$
G F \rightarrow(1 / 4)\left(G_{n+1}^{m+1} F_{n}^{m}+G_{n}^{m} F_{n+1}^{m+1}+G_{n}^{m+1} F_{n+1}^{m}+G_{n+1}^{m} F_{n}^{m+1}\right),
$$

and find the gauge invariant fully discrete CID equations

$$
\begin{aligned}
& G_{n+1}^{m+1} F_{n}^{m}-G_{n}^{m+1} F_{n+1}^{m}-G_{n+1}^{m} F_{n}^{m+1}+G_{n}^{m} F_{n+1}^{m+1} \\
& \quad=(\delta \varepsilon / 2)\left(G_{n+1}^{m+1} F_{n}^{m}+G_{n}^{m} F_{n+1}^{m+1}+G_{n}^{m+1} F_{n+1}^{m}+G_{n+1}^{m} F_{n}^{m+1}\right), \\
& \left(1 / \varepsilon^{2}\right)\left(F_{n}^{m+1} F_{n}^{m-1}-\left(F_{n}^{m}\right)^{2}\right)=G_{n}^{m} G_{n}^{m} .
\end{aligned}
$$

We introduce the auxiliary variable $\Gamma_{n}^{m}$ defined by

$$
\Gamma_{n}^{m}=\frac{F_{n}^{m+1} F_{n+1}^{m}}{F_{n}^{m} F_{n+1}^{m+1}} .
$$

It is easy to check that $\Gamma_{n}^{m}$ satisfies the identity

$$
\Gamma_{n}^{m}=\frac{F_{n}^{m+1} F_{n}^{m-1}}{\left(F_{n}^{m}\right)^{2}} \frac{\left(F_{n+1}^{m}\right)^{2}}{F_{n+1}^{m+1} F_{n+1}^{m-1}} \Gamma_{n}^{m-1} .
$$

Let $G_{n}^{m}=r_{n}^{m} F_{n}^{m}$. The eqs. (4.6)-(4.7) are then transformed into the ordinary nonlinear form

$$
\begin{aligned}
& r_{n+1}^{m+1}+r_{n}^{m}-\left(r_{n}^{m+1}+r_{n+1}^{m}\right) \Gamma_{n}^{m}=\frac{\delta \varepsilon}{2}\left[r_{n+1}^{m+1}+r_{n}^{m}+\left(r_{n}^{m+1}+r_{n+1}^{m}\right) \Gamma_{n}^{m+1}\right], \\
& \Gamma_{n}^{m}=\frac{1+\left(\varepsilon r_{n}^{m}\right)^{2}}{1+\left(\varepsilon r_{n+1}^{m}\right)^{2}} \Gamma_{n}^{m-1} .
\end{aligned}
$$

The fully discrete CID equations are the 1-component form of the coupled discrete Klein-Gordon (sine-Gordon) equation [13]. Let $\varepsilon=\delta=1$. The one-soliton solution for fully discrete CID Eqs. (4.6)-(4.7) is given by

$$
G_{n}^{m}=c p^{m} q^{n}, \quad F_{n}^{m}=1+\frac{p^{2 m} q^{2 n}}{\left(p-p^{-1}\right)^{2}},
$$

where $p, q$ and $c$ are arbitrary constants and satisfy the dispersion relation $q=\frac{3 p-1}{p-3}$. The two-soliton solution reads

$$
\begin{aligned}
F_{n}^{m}= & 1+a\left(1,1^{*}\right) \exp \left(2 \eta_{1}\right)+a\left(2,2^{*}\right) \exp \left(2 \eta_{2}\right) \\
& +\left[a\left(1,2^{*}\right)+a\left(2,1^{*}\right)\right] \exp \left(\eta_{1}+\eta_{2}\right)+a\left(1,2,1^{*}, 2^{*}\right) \exp \left(2 \eta_{1}+2 \eta_{2}\right), \\
G_{n}^{m}= & \exp \left(\eta_{1}\right)+\exp \left(\eta_{2}\right)+a\left(1,2,1^{*}\right) \exp \left(2 \eta_{1}+\eta_{2}\right)+a\left(1,2,2^{*}\right) \exp \left(\eta_{1}+2 \eta_{2}\right),
\end{aligned}
$$

where $\exp \left(\eta_{i}\right)$ and the coefficients are defined by

$$
\begin{aligned}
& \exp \left(\eta_{i}\right)=c_{i} p_{i}^{m} q_{i}^{n}, \quad q_{i}=\frac{3 p_{i}-1}{p_{i}-3}, \\
& a(i, j)=\frac{\left(p_{i}-p_{j}\right)^{2}}{p_{i} p_{j}}, \\
& a\left(i, j^{*}\right)=\frac{p_{i} p_{j}}{\left(p_{i} p_{j}-1\right)^{2}}, \\
& a\left(i^{*}, j^{*}\right)=\frac{\left(p_{i}-p_{j}\right)^{2}}{p_{i} p_{j}} .
\end{aligned}
$$


It is easy to verify the three-soliton solutions for the fully discrete CID eqs. (4.6)-(4.7) in the form (2.38)-(2.39) with the coefficients (4.16)-(4.18). Similar as the discussion in the previous semidiscrete cases, we present here the $N$-soliton solutions

$$
\begin{aligned}
& F_{n}^{m}=\sum_{\mu=0,1}^{(e)} \exp \left[\sum_{j=1}^{2 N} \mu_{j} \eta_{j}+\sum_{1 \leq k<l}^{2 N} \mu_{k} \mu_{l} A_{k l}\right], \\
& G_{n}^{m}=\sum_{v=0,1}^{(o)} \exp \left[\sum_{j=1}^{2 N} v_{j} \eta_{j}+\sum_{1 \leq k<l}^{2 N} v_{k} v_{l} A_{k l}\right],
\end{aligned}
$$

where

$$
\begin{aligned}
& \exp \left(\eta_{j}\right)=\exp \left(\eta_{N+j}\right)=c_{j} p_{j}^{m} q_{j}^{n}, \quad q_{j}=\frac{3 p_{j}-1}{p_{j}-3}, \quad j=1,2, \cdots, N, \\
& \exp \left(A_{k l}\right)=\frac{\left(p_{k}-p_{l}\right)^{2}}{p_{k} p_{l}}, k<l=2,3, \cdots, N \\
& \exp \left(A_{k, N+l}\right)=\frac{p_{k} p_{l}}{\left(p_{k} p_{l}-1\right)^{2}}, k, l=1,2, \cdots, N, \\
& \exp \left(A_{N+k, N+l}\right)=\frac{\left(p_{k}-p_{l}\right)^{2}}{p_{k} p_{l}}, k<l=2,3, \cdots, N .
\end{aligned}
$$

Here $c_{j}, p_{j}, q_{j}$ are all real parameters. The summations $\sum_{\mu=0,1}^{(e)}$ and $\sum_{v=0,1}^{(o)}$ satisfy the condition (2.47) and (2.48) respectively.

\section{Discrete analogues by 2-reduction}

Since the relationship between the sine-Gordon equation and the CID equations, in semi- and fully discrete analogues of the CID equations, there are also connections with semi- and fully discrete sine-Gordon equations. In ref. [5], authors derived the discrete analogues and Casorati determinant solutions of short pulse equation by 2-reduction of the bilinear two-dimensional Toda lattice (2DTL) equations. Following the idea [5], in this section we use the reduction technique to deduce discrete analogues and Casorati determinant solutions of the CID equations.

It is known that by setting $q=q_{0} \cos \phi, r=\frac{1}{2} \phi_{t}$, the CID equations

$$
\begin{aligned}
& q_{t}+2 r r_{x}=0, \\
& r_{x t}-2 q r=0,
\end{aligned}
$$

are transformed into the sine-Gordon equation

$$
\phi_{x t}=2 q_{0} \sin \phi .
$$

We take $q_{0}=1 / 2$ for simplicity in the following discussion. As is shown $[11,12]$, upon the dependent variable transformation

$$
\phi(x, t)=2 \mathrm{i} \ln \frac{F^{*}(x, t)}{F(x, t)}
$$


the sine-Gordon equation (5.3) leads to the bilinear form:

$$
\begin{aligned}
& F F_{x t}-F_{x} F_{t}=\frac{1}{4}\left(F^{2}-F^{* 2}\right), \\
& F^{*} F_{x t}^{*}-F_{x}^{*} F_{t}^{*}=\frac{1}{4}\left(F^{* 2}-F^{2}\right),
\end{aligned}
$$

where $F^{*}$ is the complex conjugate of $F$. Henceforth, the solutions of the CID equations are obtained by $F$ and $F^{*}$ through the dependent variable transformation

$$
q=\frac{1}{2} \cos \left(2 \mathrm{i} \ln \frac{F^{*}(x, t)}{F(x, t)}\right), \quad r=\frac{1}{2} \frac{\partial}{\partial t} \phi=\left(\mathrm{i} \ln \frac{F^{*}(x, t)}{F(x, t)}\right)_{t} .
$$

It has been shown in [5] that the bilinear equations (5.4) and (5.5) are actually obtained as the 2 -reduction of the 2DTL equations:

$$
\frac{1}{2} D_{X} D_{T} \tau_{n} \cdot \tau_{n}=\tau_{n}^{2}-\tau_{n+1} \tau_{n-1},
$$

i.e.,

$$
\tau_{n} \frac{\partial^{2} \tau_{n}}{\partial X \partial T}-\frac{\partial \tau_{n}}{\partial X} \frac{\partial \tau_{n}}{\partial T}=\tau_{n}^{2}-\tau_{n+1} \tau_{n-1}
$$

Applying the 2-reduction $\tau_{n-1}=\alpha \tau_{n+1}$ ( $\alpha$ is a constant), we get

$$
\tau_{n} \frac{\partial^{2} \tau_{n}}{\partial X \partial T}-\frac{\partial \tau_{n}}{\partial X} \frac{\partial \tau_{n}}{\partial T}=\tau_{n}^{2}-\tau_{n+1}^{2}
$$

where the gauge transformation $\tau_{n} \rightarrow \alpha^{\frac{n}{2}} \tau_{n}$ is used. Letting $f=\tau_{0}$ and $\bar{f}=\tau_{1}$, we have

$$
\begin{aligned}
& f f_{X T}-f_{X} f_{T}=f^{2}-\bar{f}^{2}, \\
& \bar{f} \bar{f}_{X T}-\bar{f}_{X} \bar{f}_{T}=\bar{f}^{2}-f^{2},
\end{aligned}
$$

Under the independent variable transformation $x=2 X, t=2 T$, we obtain

$$
\begin{aligned}
& f f_{x t}-f_{x} f_{t}=\frac{1}{4}\left(f^{2}-\bar{f}^{2}\right), \\
& \bar{f} \bar{f}_{x t}-\bar{f}_{x} \bar{f}_{t}=\frac{1}{4}\left(\bar{f}^{2}-f^{2}\right),
\end{aligned}
$$

which are bilinear form of the CID equations.

Next we give the Casorati determinant ( $N$-soliton) solution of the CID equation. The Casorati determinant solution of the 2TDL equation is of the form

$$
\tau_{n}(X, T)=\left|\psi_{i}^{(n+j-1)}(X, T)\right|_{1 \leq i, j \leq N},
$$

where $\psi_{i}^{(n)}(X, T)$ satisfies linear dispersion relations

$$
\frac{\partial \psi_{i}^{(n)}}{\partial X}=\psi_{i}^{(n+1)}, \quad \frac{\partial \psi_{i}^{(n)}}{\partial T}=\psi_{i}^{(n-1)} .
$$

For example, a particular choice of $\psi_{i}^{(n)}(X, T)$

$$
\psi_{i}^{(n)}(X, T)=c_{i} p_{i}^{n} e^{p_{i} X+\frac{1}{p_{i}} T+\eta_{0 i}}+d_{i} q_{i}^{n} e^{q_{i} X+\frac{1}{q_{i}} T+\eta_{0 i}^{\prime}},
$$


with $c_{i}$ and $d_{i}$ being constants, satisfies the linear dispersion relations and gives the $N$-soliton solutions.

Applying the 2-reduction $q_{i}=-p_{i}$ and the change of variables $x=2 X$ and $t=2 T$, we obtain the determinant solution of bilinear equations (5.12)-(5.13):

$$
\begin{aligned}
& f(x, t)=\tau_{0}(x, t), \quad \bar{f}(x, t)=\tau_{1}(x, t), \\
& \tau_{n}(x, t)=\left|\psi_{i}^{(n+j-1)}(x, t)\right|_{1 \leq i, j \leq N},
\end{aligned}
$$

where

$$
\psi_{i}^{(n)}(x, t)=c_{i} p_{i}^{n} e^{\frac{1}{2} p_{i} x+\frac{1}{2 p_{i}} t+\eta_{0 i}}+d_{i}\left(-p_{i}\right)^{n} e^{-\frac{1}{2} p_{i} x-\frac{1}{2 p_{i}} t+\eta_{0 i}^{\prime}} .
$$

Let us introduce $\alpha$ and $\beta$ such that $F^{*}=\alpha \bar{f}$ and $F=\beta f$, where $F$ and $F^{*}$ are complex conjugate of each other. Note that a set of $F$ and $F^{*}$ gives solutions of the CID equations as well as a set of $f$ and $\bar{f}$ because of

$$
r=\frac{1}{2} \frac{\partial}{\partial t}\left(2 \mathrm{i} \ln \frac{F^{*}}{F}\right)=\frac{1}{2} \frac{\partial}{\partial t}\left(2 \mathrm{i} \ln \frac{\alpha \bar{f}}{\beta f}\right)=\frac{1}{2} \frac{\partial}{\partial t}\left(2 \mathrm{i} \ln \frac{\bar{f}}{f}\right) .
$$

By choosing phase constants appropriately, the functions $f$ and $\bar{f}$ can be made to be complex conjugate of each other to keep the reality and regularity of $r$. For example, the following choice

$$
\psi_{i}^{(n)}(x, t)=p_{i}^{n} e^{\frac{1}{2} p_{i} x+\frac{1}{2 p_{i}} t+\eta_{0 i}-\mathrm{i} \pi / 4}+\left(-p_{i}\right)^{n} e^{-\frac{1}{2} p_{i} x-\frac{1}{2 p_{i}} t+\eta_{0 i}^{\prime}+\mathrm{i} \pi / 4}
$$

guarantees the reality and regularity of the solution.

Summarizing the above results, the determinant solution of the CID equation is given by

$$
\begin{aligned}
& q=\frac{1}{2} \cos \left(2 \mathrm{i} \ln \frac{\bar{f}(x, t)}{f(x, t)}\right), \quad r=\frac{1}{2} \frac{\partial}{\partial t} \phi=\frac{\partial}{\partial t}\left(\mathrm{i} \ln \frac{\bar{f}(x, t)}{f(x, t)}\right), \\
& f(x, t)=\tau_{0}(x, t), \quad \bar{f}(x, t)=\tau_{1}(x, t), \\
& \tau_{n}(x, t)=\left|\psi_{i}^{(n+j-1)}(x, t)\right|_{1 \leq i, j \leq N},
\end{aligned}
$$

where

$$
\psi_{i}^{(n)}(x, t)=p_{i}^{n} e^{\frac{1}{2} p_{i} x+\frac{1}{2 p_{i}} t+\eta_{0 i}-\mathrm{i} \pi / 4}+\left(-p_{i}\right)^{n} e^{-\frac{1}{2} p_{i} x-\frac{1}{2 p_{i}} t+\eta_{0 i}^{\prime}+\mathrm{i} \pi / 4} .
$$

Consider the following Casorati determinant:

$$
\tau_{n}(k, T)=\left|\psi_{i}^{(n+j-1)}(k, T)\right|_{1 \leq i, j \leq N},
$$

where $\psi_{i}^{(n)}$ satisfies the dispersion relations

$$
\begin{aligned}
& \triangle_{k} \psi_{i}^{(n)}=\psi_{i}^{(n+1)}, \\
& \partial_{T} \psi_{i}^{(n)}=\psi_{i}^{(n-1)},
\end{aligned}
$$

where $\triangle_{k}$ is the backward difference operator with the spacing constant $a$ :

$$
\triangle_{k} f(k)=\frac{f(k)-f(k-1)}{a} .
$$


One can choose

$$
\psi_{i}^{(n)}(k, T)=c_{i} p_{i}^{n}\left(1-a p_{i}\right)^{-k} e^{\frac{1}{p_{i}} T+\xi_{i 0}}+d_{i} q_{i}^{n}\left(1-a q_{i}\right)^{-k} e^{\frac{1}{q_{i}} T+\eta_{i 0}},
$$

which automatically satisfies the dispersion relation (5.24) and (5.25). The above Casorati determinant satisfies the bilinear form of the semi-discrete 2DTL equation

$$
\left(\frac{1}{a} D_{T}-1\right) \tau_{n}(k+1) \cdot \tau_{n}(k)+\tau_{n+1}(k+1) \cdot \tau_{n-1}(k)=0
$$

Applying the 2-reduction

$$
q_{i}=-p_{i}
$$

and letting

$$
f_{k}=\tau_{0}(k), \quad \bar{f}_{k}=\tau_{1}(k)=\left(\prod_{i=1}^{N} p_{i}^{2}\right) \tau_{-1}(k)
$$

we obtain

$$
\begin{aligned}
& \frac{1}{a} D_{T} f_{k+1} \cdot f_{k}-f_{k+1} f_{k}+\bar{f}_{k+1} \bar{f}_{k}=0, \\
& \frac{1}{a} D_{T} \bar{f}_{k+1} \cdot \bar{f}_{k}-\bar{f}_{k+1} \bar{f}_{k}+f_{k+1} f_{k}=0,
\end{aligned}
$$

where the gauge transformation $\tau_{n} \rightarrow\left(\prod_{i=1}^{N} p_{i}\right)^{n} \tau_{n}$ is used. Under the change of independent variable $t=2 T$, from (5.29)-(5.30) we can obtain

$$
\frac{2}{a}\left(\left(\ln \frac{\bar{f}_{k+1}}{\bar{f}_{k}}\right)_{t}-\left(\ln \frac{f_{k+1}}{f_{k}}\right)_{t}\right)=\frac{\bar{f}_{k+1} \bar{f}_{k}}{f_{k+1} f_{k}}-\frac{f_{k+1} f_{k}}{\bar{f}_{k+1} \bar{f}_{k}}
$$

One can check the deducing details in [5]. Introducing the dependent variable transformation $\phi_{k}(t)=2 \mathrm{i} \ln \frac{\bar{f}_{k}(s)}{f_{k}(s)}$, one arrives at

$$
\frac{1}{2 a}\left(\frac{\mathrm{d} \phi_{k+1}}{\mathrm{~d} t}-\frac{\mathrm{d} \phi_{k}}{\mathrm{~d} t}\right)=\sin \left(\frac{\phi_{k+1}+\phi_{k}}{2}\right),
$$

which is nothing but an integrable semi-discretization of the sine-Gordon equation. By introducing the variable transformation

$$
\begin{aligned}
& r_{k}=\frac{1}{2} \frac{\mathrm{d} \phi_{k}}{\mathrm{~d} t}=\frac{\mathrm{d}}{\mathrm{d} t}\left(\mathrm{i} \ln \frac{\bar{f}_{k}(s)}{f_{k}(s)}\right), \\
& q_{k}=\cos \left(\frac{\phi_{k+1}+\phi_{k}}{2}\right)=\frac{1}{2}\left(\frac{\bar{f}_{k+1} \bar{f}_{k}}{f_{k+1} f_{k}}+\frac{f_{k+1} f_{k}}{\bar{f}_{k+1} \bar{f}_{k}}\right),
\end{aligned}
$$

and together with the fact

$$
\cos ^{2}\left(\frac{\phi_{k+1}+\phi_{k}}{2}\right)+\sin ^{2}\left(\frac{\phi_{k+1}+\phi_{k}}{2}\right)=1
$$


we can obtain an integrable semi-discrete analogue of the CID equations

$$
\begin{aligned}
& \left(r_{k+1}-r_{k}\right)^{2}=a^{2}\left(1-q_{k}^{2}\right), \\
& \frac{\mathrm{d} q_{k}}{\mathrm{~d} t}=\frac{r_{k}^{2}-r_{k+1}^{2}}{a} .
\end{aligned}
$$

From the construction of the semi-discrete analogue, we know that the semi-discrete analogue of the CID equations has the following Casorati determinant solution:

$$
\begin{aligned}
& r_{k}(t)=\frac{\mathrm{d}}{\mathrm{d} t}\left(\mathrm{i} \ln \frac{\bar{f}_{k}}{f_{k}}\right), \\
& f_{k}(t)=\tau_{0}(k, t), \quad \bar{f}_{k}(t)=\tau_{1}(k, t), \\
& \tau_{n}(k, t)=\left|\psi_{i}^{(n+j-1)}(k, t)\right|_{1 \leq i, j \leq N},
\end{aligned}
$$

with

$$
\psi_{i}^{(n)}(k, t)=p_{i}^{n}\left(1-a p_{i}\right)^{-k} e^{\frac{1}{2 p_{i}} t+\xi_{i 0}-\mathrm{i} \pi / 4}+\left(-p_{i}\right)^{n}\left(1+a p_{i}\right)^{-k} e^{-\frac{1}{2 p_{i}} t+\eta_{i 0}+\mathrm{i} \pi / 4} .
$$

We can rewrite the semi-discrete CID equations in an alternative version which converges to the CID equations in the continuous limit $a \rightarrow 0$. Multiplying eq. (5.37) by $2 q_{k}$, we have

$$
\frac{\mathrm{d} q_{k}^{2}}{\mathrm{~d} t}=\frac{2 q_{k}\left(r_{k}^{2}-r_{k+1}^{2}\right)}{a}
$$

A substitution of $q_{k}^{2}$ from (5.36) into equation (5.41) results to

$$
\frac{\mathrm{d}}{\mathrm{d} t} \frac{r_{k+1}-r_{k}}{a}=q_{k}\left(r_{k+1}+r_{k}\right) .
$$

In the continuous limit $a \rightarrow 0,(5.37)$ and (5.42) converge to the CID equations

$$
\begin{aligned}
& q_{t}+2 r r_{x}=0, \\
& r_{x t}-2 q r=0 .
\end{aligned}
$$

By the dependent variable transformation $1-\frac{q_{n+1}-q_{n}}{\varepsilon} \rightarrow q_{n}$, the previous semi-discrete CID equations (2.17) and (2.18) turn into (5.37) and (5.42). Considering the symmetry of $x$ and $t$ in the sine-Gordon equation, semi-discrete analogue of $t$ part can be completed similarly.

To construct a full-discrete analogue of the CID equations, we introduce one more discrete variable $l$ which corresponds to the discrete time variable. It is known that the bilinear equations

$$
\left(\frac{2}{a} D_{s}-1\right) \tau_{n}(k+1, l) \cdot \tau_{n}(k, l)+\tau_{n+1}(k+1, l) \tau_{n-1}(k, l)=0,
$$

and

$$
\left(2 b D_{s}-1\right) \tau_{n}(k, l+1) \cdot \tau_{n+1}(k, l)+\tau_{n}(k, l) \tau_{n+1}(k, l+1)=0,
$$

have the Casorati determinant solution

$$
\tau_{n}(k, l)=\left|\psi_{i}^{(n+j-1)}(k, l)\right|_{1 \leq i, j \leq N},
$$


with

$$
\psi_{i}^{(n)}=c_{i} p_{i}^{n}\left(1-a p_{i}\right)^{-k}\left(1-\frac{b}{p_{i}}\right)^{-l} e^{\frac{1}{2 p_{i}} s+\xi_{i 0}}+d_{i} q_{i}^{n}\left(1-a q_{i}\right)^{-k}\left(1-\frac{b}{q_{i}}\right)^{-l} e^{\frac{1}{2 q_{i}} s+\eta_{i 0}} .
$$

Applying the 2-reduction $\tau_{n-1}=\left(\prod_{i=1}^{N} p_{i}^{2}\right)^{-1} \tau_{n+1}$, i.e. adding constraint $q_{i}=-p_{i}$ to the $N$-soliton solution, one obtains

$$
\left(\frac{2}{a} D_{s}-1\right) \tau_{n}(k+1, l) \cdot \tau_{n}(k, l)+\tau_{n+1}(k+1, l) \tau_{n+1}(k, l)=0,
$$

and

$$
\left(2 b D_{s}-1\right) \tau_{n}(k, l+1) \cdot \tau_{n+1}(k, l)+\tau_{n}(k, l) \tau_{n+1}(k, l+1)=0,
$$

where the gauge transformation $\tau_{n} \rightarrow\left(\prod_{i=1}^{N} p_{i}\right)^{n} \tau_{n}$ is used. Letting

$$
f_{k, l}=\tau_{0}(k, l), \quad \bar{f}_{k, l}=\tau_{1}(k, l),
$$

and introducing

$$
r_{k, l}=\left(\mathrm{i} \ln \frac{\bar{f}_{k, l}}{f_{k, l}}\right)_{s}
$$

together with auxiliary variable

$$
v_{k, l}=k a-\left(\ln \bar{f}_{k, l} f_{k, l}\right)_{s}
$$

from (5.47) and (5.48), we find the following relations

$$
\begin{aligned}
& \left(r_{k+1, l}-r_{k, l}\right)^{2}=a^{2}\left(1-q_{k, l}^{2}\right), \\
& \left(r_{k, l+1}+r_{k, l}\right)^{2}=\frac{1}{b^{2}}-\left(v_{k, l+1}-v_{k, l}+\frac{1}{b}\right)^{2},
\end{aligned}
$$

where $q_{k, l}=\frac{v_{k+1, l}-v_{k, l}}{a}$. Substituting $k=k+1$ into (5.52) and subtracting each other, we obtain

$$
\begin{aligned}
& \left(r_{k, l+1}+r_{k, l}\right)^{2}-\left(r_{k+1, l+1}+r_{k+1, l}\right)^{2} \\
= & \left(v_{k+1, l+1}-v_{k, l}\right)^{2}-\left(v_{k, l+1}-v_{k, l}\right)^{2}+\frac{2 a}{b}\left(q_{k, l+1}-q_{k, l}\right),
\end{aligned}
$$

which converges to

$$
\frac{\mathrm{d} q_{k}}{\mathrm{~d} t}=\frac{r_{k}^{2}-r_{k+1}^{2}}{a}
$$

in the continuous limit $b \rightarrow 0$. Hence eqs. (5.51) and (5.52) constitute an integrable fulldiscretization of the CID equation. 
From the construction above, the determinant solution of the full-discrete CID equation is

$$
\begin{aligned}
& r_{k, l}=\mathrm{i}\left(\frac{\bar{g}_{k, l}}{\bar{f}_{k, l}}-\frac{g_{k, l}}{f_{k, l}}\right)=\frac{\mathrm{d}}{\mathrm{d} t}\left(\mathrm{i} \ln \frac{\bar{f}_{k, l}}{\bar{f}_{k, l}}\right), \\
& q_{k, l}=\frac{1}{2}\left(\frac{\bar{f}_{k+1, l} \bar{f}_{k, l}}{f_{k+1, l} f_{k, l}}+\frac{f_{k+1, l} f_{k, l}}{\bar{f}_{k+1, l} \bar{f}_{k, l}}\right), \\
& f_{k, l}=\tau_{0}(k, l), \quad \bar{f}_{k, l}=\tau_{1}(k, l), \\
& g_{k, l}=\rho_{0}(k, l), \quad \bar{g}_{k, l}=\rho_{1}(k, l), \\
& \tau_{n}(k, l)=\left|\begin{array}{cccc}
\psi_{1}^{(n)}(k, l) & \psi_{1}^{(n+1)}(k, l) & \cdots & \psi_{1}^{(n+N-1)}(k, l) \\
\psi_{2}^{(n)}(k, l) & \psi_{2}^{(n+1)}(k, l) & \cdots & \psi_{2}^{(n+N-1)}(k, l) \\
\vdots & \vdots & & \vdots \\
\psi_{N}^{(n)}(k, l) & \psi_{N}^{(n+1)}(k, l) & \cdots & \psi_{N}^{(n+N-1)}(k, l)
\end{array}\right| \\
& \rho_{n}(k, l)=\left|\begin{array}{cccc}
\psi_{1}^{(n-1)}(k, l) & \psi_{1}^{(n+1)}(k, l) & \cdots & \psi_{1}^{(n+N-1)}(k, l) \\
\psi_{2}^{(n-1)}(k, l) & \psi_{2}^{(n+1)}(k, l) & \cdots & \psi_{2}^{(n+N-1)}(k, l) \\
\vdots & \vdots & & \vdots \\
\psi_{N}^{(n-1)}(k, l) & \psi_{N}^{(n+1)}(k, l) & \cdots & \psi_{N}^{(n+N-1)}(k, l)
\end{array}\right|,
\end{aligned}
$$

with

$$
\psi_{i}^{(n)}=p_{i}^{n}\left(1-a p_{i}\right)^{-k}\left(1-\frac{b}{p_{i}}\right)^{-l} e^{\frac{1}{2 p_{i}} s+\xi_{i 0}-\mathrm{i} \pi / 4}+\left(-p_{i}\right)^{n}\left(1+a p_{i}\right)^{-k}\left(1+\frac{b}{p_{i}}\right)^{-l} e^{-\frac{1}{2 p_{i}} s+\eta_{i 0}+\mathrm{i} \pi / 4},
$$

where the phase constants $\pm \mathrm{i} \pi / 4$ play the role of keeping the reality of the solution and $s$ is an auxiliary parameter.

\section{Conclusion and discussion}

Integrability and $N$-soliton solution of the semi-discrete sine-Gordon equation

$$
\phi_{n+1, t}+\phi_{n, t}=\sin \phi_{n+1}-\sin \phi_{n}
$$

have been shown in $[23,27]$ and [25] respectively. Since the CID system shares the bilinear form with sine-Gordon equation, it is natural to consider the relation between the semi-discrete CID eqs. (2.17)-(2.18) (or (5.37) and (5.42)) and the semi-discrete sine-Gordon eq. (6.1).

It is known that coupled forms of integrable models can be obtained from the bilinear equations. See for example the KdV equation [30], sine-Gordon equation [16], modified KdV equation [16], vector Ito equation [14], nonlinear Schrödinger equation [24], NNV equation [32]. The coupled form of the CID equations can hence also be given

$$
\begin{aligned}
& D_{t}^{2} F \cdot F=2 \sum_{k=1}^{N} G_{k}^{2}, \\
& \left(D_{x} D_{t}-2\right) G_{k} \cdot F=0 .
\end{aligned}
$$


They can be transformed into the nonlinear form

$$
\begin{aligned}
& u_{t}=\sum_{k=1}^{N} r_{k}^{2}, \\
& \left(r_{k}\right)_{x t}+2 r_{k}\left(u_{x}-1\right)=0,
\end{aligned}
$$

by the dependent variable transformation $u=(\ln F)_{t}, G_{k}=r_{k} F$. A further extension of the coupled form is

$$
\begin{aligned}
& D_{t}^{2} F \cdot F=2 \sum_{j, k=1}^{N} c_{j, k} G_{j} G_{k}, \\
& \left(D_{x} D_{t}-2\right) G_{j} \cdot F=0,
\end{aligned}
$$

where $c_{j, k}$ are arbitrary coupling constants. With the transformation $G_{j}=r_{j} F, u=2(\ln F)_{t}$, the general coupled CID eqs. (6.6)-(6.7) are mapped into the ordinary nonlinear form

$$
\begin{aligned}
& u_{t}=\sum_{j, k=1}^{N} c_{j, k} r_{j} r_{k}, \\
& \left(r_{j}\right)_{x t}+2 r_{j}\left(u_{x}-1\right)=0 .
\end{aligned}
$$

From the bilinear integrable discretization approach, discrete version of these coupled CID equations can also be obtained [13].

To summarize, we have presented here two semi-discrete integrable versions and one fully discrete integrable version for the CID equations and have derived their soliton solutions. Based on the results obtained, it would be natural to further consider other integrable properties such as the determinant structure of the $\mathrm{N}$-soliton solutions for these semi-discrete and fully discrete integrable lattice.

\section{Acknowledgements}

Authors are grateful to anonymous referee for pointing out relevant literature and helpful suggestion in improving the original manuscript. The work of L.V. is supported by a grant from the National Science and Engineering Research Council (NSERC) of Canada. G. Yu acknowledges a postdoctoral fellowship from the Mathematical Physics Laboratory of the CRM. He is also supported by the National Natural Science Foundation of China (Grant no.10901105) and Chenguang Program (No.09CG08) sponsored by Shanghai Municipal Education Commission and Shanghai Educational Development Foundation.

\section{References}

[1] M. J. Ablowitz and J. F. Ladik, Stud. Appl. Math. 55 (1976) 213; 57 (1977) 1; J. Math. Phys. 17 (1976) 1011.

[2] M. J. Ablowitz and H. Segur, Solitons and the Inverse Scattering Transform, SIAM Studies in Applied Mathematics, 4. Society for Industrial and Applied Mathematics (SIAM), Philadelphia, Pa., 1981

[3] T. Alagesan, Y. Chung, K. Nakkeeran, Bäcklund transformation and soliton solutions for the coupled dispersionless equations, Chaos, Solitons and Fractals. 21 (2004) 63-67.

[4] T. Alageasn and K. Porsezian, Singularity Structure Analysis and Hirota's Bilinearisation of the Coupled Integrable Dispersionless Equations, Chaos, Soliton and Fractals. 8 (1997) 1645. 
[5] B.F. Feng, K. Maruno, Y. Ohta, Integrable discretizations of the short pulse equation, J. Phys. A. 43 (2010) 085203.

[6] R. Hirota, Nonlinear partial difference equations I, J. Phys. Soc. Jpn. 43 (1977) 1424-1433.

[7] R. Hirota, Nonlinear partial difference equations II; Discrete time Toda equations, J. Phys. Soc. Jpn. 43 (1977) 2074-2078.

[8] R. Hirota, Nonlinear partial difference equations III; Discrete sine-Gordon equation, J. Phys. Soc. Jpn. 43 (1977) 2079-2086.

[9] R. Hirota, Discrete analogue of a generalized Toda equation, J. Phys. Soc. Jpn. 50 (1981) 3785-3791.

[10] R. Hirota, Exact envelope-soliton solutions of a nonliear wave equation, J. Math. Phys. 14 (1973), 805-809.

[11] R. Hirota, Exact Solution of the sine-Gordon Equation for Multiple Collisions of Solitons, J. Phys. Soc. Jpn. 33 (1972) 1459-1463.

[12] R.Hirota, 2004 The direct method in soliton theory (Cambridge: Cambridge University Press)

[13] R. Hirota, Discretization of coupled soliton equations, Bilinear Integrable Systems: From Classical to Quantum, Continuous to Discrete, NATO Sci. Ser. II Math. Phys. Chem., 201, Springer, Dordrecht, (2006) 113.

[14] R. Hirota, X.B. Hu, X.Y. Tang, A vector potential KdV equation and vector Ito equation: Soliton solutions, bilinear Bäklund transformation and Lax pairs.J. Math. Anal. Appl. 288 (2003) 326-348.

[15] R. Hirota and S. Tsujimoto, Note on "New coupled integrable dispersionless equation", J.Phys.Soc.Jpn. 63 (1994) 3533.

[16] R. Hirota and Y. Ohta, Hierarchies of coupled Soliton equations. I,J. Phys. Soc. Jpn. 60 (1991) 798-809.

[17] X.B. Hu, G.F. Yu, Integrable discretizations of the (2+1)-dimensional sinh-Gordon equation. J. Phys. A. 40 (2007), 12645-12659.

[18] X.B. Hu, G.F. Yu, Integrable semi-discretizations and full-discretization of the two-dimensional Leznov lattice. J. Difference Equ. Appl. 15 (2009), 233-252.

[19] H. Kakuhata and K. Konno, A generalization of coupled integrable, dispersionless system.J. Phys. Soc. Jpn. 65 (1996) 340-341.

[20] H. Kakuhata and K. Konno, Loop soliton solutions of string interacting with external field. J. Phys. Soc. Jpn. 68 (1999) 757-762.

[21] K. Konno and H. Oono, New coupled integrable dispersionless equation, J. Phys. Soc. Jpn. 63 (1994) 377-378.

[22] K. Konno and H. Oono, Reply to Note on "New coupled integrable dispersionless equation",J.Phys.Soc.Jpn. 63 (1994) 3534.

[23] D. Levi, O. Ragnisco, M. Bruschi, Extension of the Zakharov-Shabat generalized inverse method to solve differential-difference and difference-difference equations. Nuovo Cimento A 58 (1980), 56-66.

[24] S. V. Manakov, On the theory of two-dimensional stationary self-focusing of electromagnetic waves, Soviet Phy. JETP. 38 (1974) 248-253.

[25] K. Narita, Discontinuous N-soliton solution for semidiscrete sine-Gordon equation. J. Phys. Soc. Jpn. 57 (1988), 2679-2684.

[26] Y. Ohta, K. Maruno, B.F Feng, An integrable semi-discretization of the Camassa-Holm equation and its determinant solution, J. Phys. A. 41 (2008) 355205.

[27] L. Pilloni, D. Levi, The inverse scattering transform for solving the discrete sine-Gordon equation. Phys. Lett. A. 92 (1982) 5-8.

[28] Y. Suris, The Problem of Integrable Discretization: Hamiltonian Approach (Birkhäuser, Basel, Switerland, 2003).

[29] P. Wang, B. Tian (etc.), Conservation Laws and Analytic Soliton Solutions for Coupled Integrable Dispersionless Equations with Symbolic Computation. Commun. Theor. Phys. 54 (2010) 687-696.

[30] T. Yoneyama, Interacting Korteweg-de Vries equations and attractive Soliton interaction, Progr. Theor. Phys. 72 (1984), 1081-1088.

[31] X.L. Yong, Y.F. Chen, Soliton solutions to Coupled Integrable Dispersionless equations. Commun. Theor. Phys. 50 (2008) 43-47. 
[32] G.F. Yu, H.W. Tam, A vector asymmetrical NNV equation: Soliton solutions, bilinear Bäklund transformation and Lax pairs. J. Math. Anal. Appl. 344 (2008) 593-600.

[33] X.Q. Zhao, J.F. Lu, On integrability and algebraic sturctures of a couple integrable dispersionless equations,J. Phys. Soc. Jpn. 68 (1999) 2151-2152. 